\title{
Full-Duplex Opportunistic Relay Selection in Future Spectrum-Sharing Networks
}

\author{
Mohammad Galal Khafagy $^{\S}$, Mohamed-Slim Alouini ${ }^{\S}$, and Sonia Aïssa* \\ $\S$ King Abdullah University of Science and Technology (KAUST), Thuwal, Makkah Province, Saudi Arabia \\ * Institut National de la Recherche Scientifique (INRS-EMT), University of Quebec, Montreal, QC, Canada \\ Email: \{mohammad.khafagy, slim.alouini\}@kaust.edu.sa, aissa@emt.inrs.ca
}

\begin{abstract}
We propose and analyze the performance of fullduplex relay selection in primary/secondary spectrum-sharing networks. Contrary to half-duplex relaying, full-duplex relaying (FDR) enables simultaneous listening/forwarding at the secondary relay, thereby allowing for a higher spectral efficiency. However, since the source and relay simultaneously transmit in FDR, their superimposed signal at the primary receiver should now satisfy the existing interference constraint which can considerably limit the secondary network throughput. In this regard, relay selection can offer an adequate solution to boost the secondary throughput while satisfying the imposed interference limit. We first analyze the performance of opportunistic relay selection among a cluster of full-duplex decode-and-forward relays with self-interference by deriving the exact cumulative distribution function of its endto-end signal-to-noise ratio. Second, we evaluate the end-to-end performance of relay selection with interference constraints due to the presence of a primary receiver. Finally, the presented exact theoretical findings are verified by numerical simulations.
\end{abstract}

Keywords-full-duplex relay, opportunistic relay selection, selfinterference, spectrum sharing.

\section{INTRODUCTION}

Full-duplex and cognitive radio technologies represent two promising enablers for fifth generation $(5 \mathrm{G})$ mobile communication networks [1], [2]. Such technologies, among others, show a real potential to meet the envisioned $5 \mathrm{G}$ network challenges, including higher spectral efficiency, reduced energy consumption, and better quality of user experience.

Spectrum sharing represents one network paradigm for the now celebrated cognitive radio technology in which adequate means are offered to resolve the scarcity problem of wireless resources. Also known as underlay cognitive radio, spectrum sharing allows the traffic of secondary cognitive users to coexist with that of the primary spectrum users as long as a certain interference level is not exceeded. In particular, when the secondary source and destination are spatially isolated, relay assistance becomes inevitable to establish successful communication via relay listening/forwarding, while satisfying the coexistence constraints with the primary user. Cognitive relay networks continue to draw a noticeable interest in the wireless communications community for its coverage extension capabilities under an efficient spectrum usage.

Adhering to the interference constraints in underlay networks, however, can considerably limit the secondary system throughput, especially when a single relay is leveraged to assist the communication between the secondary source and its far destination. To tackle such a challenge, relay selection was introduced to underlay networks, and it was shown to offer remarkable performance gains relative to its fixed relaying counterpart [3]. Cognitive relay selection was fairly investigated in the literature under the relaying strategies of amplifyand-forward (AF) and decode-and-forward (DF) [3]-[6].

All the aforementioned efforts considered conventional half-duplex relays. However, half-duplex relaying is known to suffer from a spectral efficiency loss when compared to direct transmission due to its time-orthogonal relay listening/forwarding. More recently, full-duplex communications experienced recent developments that brought the first fullduplex node prototypes into reality [7], [8]. These developments motivated further research in cooperative contexts where simultaneous listening/forwarding was allowed by full-duplex relaying $(F D R)$, thereby eliminating the known rate loss of half-duplex relays. Since then, recent efforts were directed to study FDR in several directions, for instance, in cognitive radio networks [9], [10] and FDR with relay selection [11], [12].

In [10], a cognitive underlay setting is studied in which a secondary system shares the spectrum with a primary system under a certain interference constraint. The secondary system comprises a source, a destination and a full-duplex DF relay. Under the aforementioned interference constraint, optimal source and relay power allocation was investigated with the objective to minimize the end-to-end outage probability. In [11], Krikidis et al. studied the outage performance of AF full-duplex relay selection with residual self-interference when no direct link exists between the source and the destination. Full-duplex relay selection was also briefly discussed for DF networks in [12]. However, only the simple case of non-fading self-interference link was considered, and the adverse effect of the interfering direct link in FDR was not accounted for.

Despite its anticipated performance merits, the incorporation of relay selection techniques into cognitive full-duplex relay networks remains untackled to the best of the authors' knowledge. In this work, we aim at bridging this gap by introducing relay selection to cognitive FDR networks and analyzing its offered performance gains. In underlay settings with FDR, the performance of the secondary users can be seriously limited due to the fact that now the superimposed source and relay interference components should satisfy the existing interference constraint which was previously imposed in half-duplex relaying on each transmitting node at a time. In this regard, relay selection techniques can offer an adequate solution to boost the secondary network throughput. We focus in this work on regenerative DF relaying. First, we derive the exact cumulative distribution function (CDF) for the end-toend signal-to-noise ratio (SNR) of opportunistic relay selection with and without a direct link. When a direct link exists between the source and its end destination, the instantaneous 


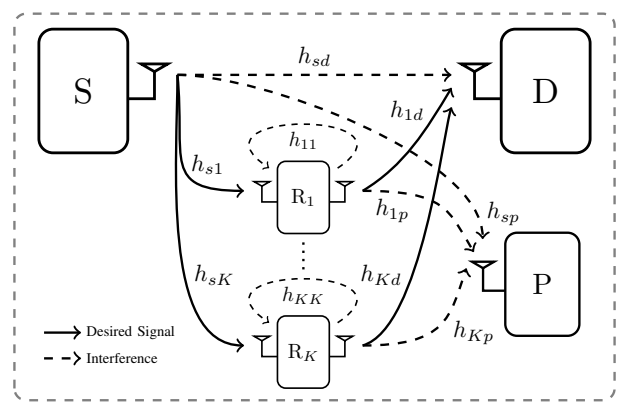

Fig. 1. Spectrum sharing network with full-duplex relaying.

SNR values of the different relaying paths become mutually dependent, which we account for in the analysis. Also, direct transmission can take place if the direct link offers higher SNR than that offered by relaying. To the best of the authors' knowledge, this opportunistic relay selection result for FDR with self-interference is presented herein for the first time. Second, the exact CDF of the end-to-end SNR is derived for opportunistic relay selection in an underlay cognitive system when an interference constraint is imposed by the primary user.

\section{Spectrum Sharing With Full-Duplex Relaying}

We consider the underlay cognitive setting depicted in Fig. 1. As shown, a secondary source (S) intends to communicate with a secondary destination (D) in the presence of a primary receiver $(\mathrm{P})$. In agreement to similar recent studies of underlay cognitive settings, for instance in [6], the primary source is assumed to be far enough that its interference effect on the secondary system can be reasonably ignored. The direct secondary source-destination link is assumed of a relatively weak gain due to distance and shadowing effects. Hence, a full-duplex relay is utilized to assist the end-toend secondary communication, taking the interference constraint on the primary receiver into account. FDR can offer higher spectral efficiency when compared to its half-duplex counterpart. However, it introduces an additional challenge in cognitive settings where higher interference levels may be experienced by the primary user of the spectrum band, due to simultaneous source/relay transmissions. Also, FDR suffers from a residual self-interference level which imposes an additional communication challenge.

In this regard, relay selection is proposed as an adequate mean to tackle and alleviate the aforementioned interference problems. Specifically, the secondary system has the additional degree of freedom to select one relay out of a cluster of $K$ full-duplex relays, $\mathcal{R}=\left\{\mathrm{R}_{1}, \mathrm{R}_{2}, \cdots, \mathrm{R}_{K}\right\}$. Relay selection is widely known to offer the same diversity order of scenarios where the transmissions of multiple cooperating relays are combined, yet it has the advantage of maintaining a simple transmitter/receiver structure. In cognitive scenarios, relay selection remains of particular payoff as it offers the ability to pick the best relay in terms of a certain performance criterion while maintaining the interference limit on the primary user.

\section{A. Channel Model}

The fading coefficient of the $i-j$ link is denoted by $h_{i j}$, for $i \in\{s, 1,2, \cdots, K\}$ and $j \in\{1,2, \cdots, K, d, p\}$, where $s, d$ and $p$ refer to the source, destination and primary nodes (Fig. 1) respectively, while $k \in\{1,2, \cdots, K\}$ denotes the relay index. Moreover, the $i-j$ link gain is denoted by $g_{i j}=\left|h_{i j}\right|^{2}$. All channels are assumed to follow a block Rayleigh fading model, where $h_{i j}$ remains constant over one block, and varies independently from one block to another following a circularly symmetric complex Gaussian distribution with zero mean and variance $\pi_{i j}$. Accordingly, $\left|h_{i j}\right|$ is Rayleigh distributed, while $g_{i j}$ follows an exponential distribution with mean $\pi_{i j}$. All channel fading gains are assumed to be mutually independent. The relays operate in a full-duplex mode where simultaneous listening/forwarding is allowed with an introduced level of loopback interference. The link gain $h_{k k}$ is assumed to represent the residual self-interference after undergoing all possible isolation and cancellation techniques, as for instance [13] and the references therein. The source and the $k^{\text {th }}$ relay powers are denoted by $P_{s}$ and $P_{k}$, respectively. Also, $n_{k}$ and $n_{d}$ denote the complex additive white Gaussian noise components at the $k^{\text {th }}$ relay and the destination, with variance $\sigma_{k}^{2}$ and $\sigma_{d}^{2}$, respectively.

As commonly assumed in the literature, for instance in [6], we assume the relays are clustered somewhere between the source and the destination. Hence, the distances among the relays are much shorter than those between the relays and the source/destination. In this setting, it is reasonable to assume the following symmetric scenario where all sourcerelay links have an average gain of $\mathbb{E}\left\{g_{s k}\right\}=\pi_{s r}$, while all relay-destination links have an average gain of $\mathbb{E}\left\{g_{k d}\right\}=\pi_{r d}$, $\forall k \in\{1,2, \cdots, K\}$. Also, all relays have the same average gain to the primary receiver, denoted as $\pi_{r p}$. Moreover, we assume that all relays have their loopback interference links with the same average gain, i.e., $\mathbb{E}\left\{g_{k k}\right\}=\pi_{r r}$. Although the analysis of asymmetric scenarios remains possible, the previous assumptions allow for simpler final expressions, and yet maintain the same diversity order of the system. Finally, we assume that $\sigma_{k}^{2}=\sigma_{d}^{2}=1$, while all transmit powers are absorbed into the channel coefficients for ease of notation.

\section{B. Signal Model}

When the $k^{\text {th }}$ relay is selected, the received signals at the $k^{\text {th }}$ relay and destination at time $t$ are given, respectively, by

$$
\begin{aligned}
& y_{k}[t]=h_{s k} x[t]+h_{k k} x[t-D]+n_{k}[t], \\
& y_{d}[t]=h_{k d} x[t-D]+h_{s d} x[t]+n_{d}[t],
\end{aligned}
$$

where $D$ is the relay transmission delay relative to that of the source in symbol times. Due to the source and relay asynchronous transmissions, the signal transmitted by the relay (source) is considered as an additional noise term at the relay (destination) as commonly treated in the related literature [13]. The received signal-to-interference-plus-noise ratio (SINR) at the $k^{\text {th }}$ relay and the destination are given respectively by

$$
\gamma_{s k}=\frac{g_{s k}}{g_{k k}+1}, \quad \gamma_{k d}=\frac{g_{k d}}{g_{s d}+1} .
$$

In an underlay setting, the introduced interference level on the primary receiver is constrained not to exceed a certain threshold, $I_{\mathrm{th}}$. Thus, when the $k^{\text {th }}$ relay is selected, the interference constraint is given by

$$
g_{s p}+g_{k p} \leq I_{\mathrm{th}} .
$$

Moreover, when no relays are selected while a direct sourcedestination link exists, the interference constraint is simply given by

$$
g_{s p} \leq I_{\mathrm{th}} .
$$




\section{PERFORMANCE ANALYSIS}

We first analyze the performance of unconstrained fullduplex relay selection, then extend to the scenario with interference constraint on the primary user.

\section{A. Relay Selection}

For exponentially distributed link gains, the CDFs of $\gamma_{s k}$ and $\gamma_{k d}$, for $0 \leq \alpha<\infty$, are given respectively as in [14] by

$F_{\gamma_{s k}}(\alpha)=1-\frac{\pi_{s r} e^{-\frac{\alpha}{\pi_{s r}}}}{\alpha \pi_{r r}+\pi_{s r}}, \quad F_{\gamma_{k d}}(\alpha)=1-\frac{\pi_{r d} e^{-\frac{\alpha}{\pi_{r d}}}}{\alpha \pi_{s d}+\pi_{r d}}$.

Moreover, conditioned on $g_{s d}=\beta \geq 0$, the CDF of $\gamma_{k d}$, also for $0 \leq \alpha<\infty$, is given by

$$
F_{\gamma_{k d} \mid g_{s d}}(\alpha \mid \beta)=1-e^{-\frac{(\beta+1) \alpha}{\pi_{r d}}}
$$

Hence, for the $k^{\text {th }}$ two-hop path, i.e., via the $k^{\text {th }}$ relay, the CDF of the end-to-end SNR is given as

$$
\begin{aligned}
F_{\gamma_{k}}(\alpha) & =\mathbb{P}\left\{\min \left\{\gamma_{s k}, \gamma_{k d}\right\} \leq \alpha\right\} \\
& =1-\left(1-F_{\gamma_{s k}}(\alpha)\right)\left(1-F_{\gamma_{k d}}(\alpha)\right) \\
& =1-\frac{\pi_{s r} \pi_{r d} e^{-\alpha\left(\frac{1}{\pi_{s r}}+\frac{1}{\pi_{r d}}\right)}}{\left(\alpha \pi_{r r}+\pi_{s r}\right)\left(\alpha \pi_{s d}+\pi_{r d}\right)} .
\end{aligned}
$$

Further, conditioned on $g_{s d}=\beta$, the SNR CDF is given by

$$
F_{\gamma_{k} \mid g_{s d}}(\alpha \mid \beta)=1-\frac{\pi_{s r}}{\alpha \pi_{r r}+\pi_{s r}} e^{-\alpha\left(\frac{1}{\pi_{s r}}+\frac{\beta+1}{\pi_{r d}}\right)} .
$$

1) Without Direct Link: When the direct secondary sourcedestination link is neglected, for instance in coverage extension scenarios where no direct link exists, the SNR CDF via the $k^{\text {th }}$ path is hence given by setting $\pi_{s d}=0$ in (10) as

$$
F_{\gamma_{k}}^{\mathrm{NDL}}(\alpha)=1-\frac{\pi_{s r}}{\alpha \pi_{r r}+\pi_{s r}} e^{-\alpha\left(\frac{1}{\pi_{s r}}+\frac{1}{\pi_{r d}}\right)} .
$$

Now, we define the set of feasible relays which satisfy the interference constraint on the primary user as $\mathcal{L}$, which contains $L$ feasible relays. When max-min relay selection, also known as opportunistic relay selection, is adopted, the CDF of the end-to-end SNR conditioned on $L$ is given by

$$
\begin{aligned}
F_{\gamma}^{\mathrm{NDL}}(\alpha \mid L) & =\mathbb{P}\left\{\max _{l \in \mathcal{L}}\left\{\gamma_{l}\right\} \leq \alpha\right\}=\prod_{l \in \mathcal{L}} F_{\gamma_{l}}^{\mathrm{NDL}}(\alpha) \\
& =\left(1-\frac{\pi_{s r} e^{-\alpha\left(\frac{1}{\pi_{s r}}+\frac{1}{\pi_{r d}}\right)}}{\alpha \pi_{r r}+\pi_{s r}}\right)^{L}
\end{aligned}
$$

where the product form in (13) comes from the mutual independence of the channel fading coefficients. Clearly, when no feasible relays exist, i.e., $L=0$, communication fails and the end-to-end SNR is exactly equal to zero. Hence, the SNR distribution is given by a unit-step function at zero SNR in this particular event as given by the above expression.

2) With Direct Link: When a direct link is available, there exists $L+1$ paths from the secondary source to the destination over which communication can take place; $L$ paths due to the cooperation of one relay with direct transmissions treated as interference, or direct transmission without relay cooperation. In this case, the CDF of the end-to-end SNR conditioned on
$L=0,1,2, \cdots, K$ is given by

$$
\begin{aligned}
F_{\gamma}^{\mathrm{DL}}(\alpha \mid L) & =\mathbb{P}\left\{\max \left\{\max _{l \in \mathcal{L}}\left\{\gamma_{l}\right\}, g_{s d}\right\} \leq \alpha\right\} \\
& =\mathbb{P}\left\{\left(\bigcap_{l \in \mathcal{L}}\left(\gamma_{l} \leq \alpha\right)\right) \cap\left(g_{s d} \leq \alpha\right)\right\} .
\end{aligned}
$$

It can be noticed that, unlike the case of no direct link, now the end-to-end SNR of the different paths, $\left\{\gamma_{l}\right\}_{l=1}^{L}$, are mutually dependent due to their dependence on the common direct-link gain random variable, $g_{s d}$. Fortunately, the $L$ cooperative paths are conditionally independent given the direct-link gain. Thus, the end-to-end SNR CDF conditioned on $L$ can be written as

$$
\begin{aligned}
F_{\gamma}^{\mathrm{DL}}(\alpha \mid L) & =\int_{\beta=0}^{\alpha} \prod_{l \in \mathcal{L}} F_{\gamma_{k} \mid g_{s d}}(\alpha \mid \beta) \frac{e^{-\frac{\beta}{\pi_{s d}}}}{\pi_{s d}} \mathrm{~d} \beta \\
& =\int_{\beta=0}^{\alpha}\left(1-\frac{\pi_{s r} e^{-\alpha\left(\frac{1}{\pi_{s r}}+\frac{\beta+1}{\pi_{r d}}\right)}}{\left(\alpha \pi_{r r}+\pi_{s r}\right)}\right)^{L} \frac{e^{-\frac{\beta}{\pi_{s d}}}}{\pi_{s d}} \mathrm{~d} \beta .
\end{aligned}
$$

Thanks to the binomial expansion in (19) shown at the top of the next page, substituting in (18) and after integration, we can reach the end-to-end SNR CDF conditioned on $L$ in (20) shown at the top of the next page.

\section{B. Underlay Cognitive Relay Selection}

There exists an interference threshold, $I_{\mathrm{th}}$, that the secondary system cannot exceed. Now, we are interested in the probability to have $L$ out of the $K$ available relays that satisfy the interference constraint $I_{\mathrm{th}}$. Let us denote this probability by $\mathcal{P}_{L}$. We first start with the no direct link scenario.

1) Without Direct Link: When the $k^{\text {th }}$ relay is active, the interference constraint is given by (4). Unlike half-duplex cognitive relay settings, full-duplex operation causes a superposition of the source and relay interference at the primary receiver. Since the source interference is a common random variable when any of the $K$ relays is selected, the superimposed interference signals are correlated. However, they are conditionally independent given $g_{s p}$. Conditioned on $g_{s p}=\beta \geq 0$, the probability that the $k^{\text {th }}$ relay is feasible is given by

$$
F_{g_{k} \mid g_{s p}}\left(I_{\mathrm{th}} \mid \beta\right)= \begin{cases}1-e^{-\frac{I_{\mathrm{th}}-\beta}{\pi_{r p}}}, & \text { if } I_{\mathrm{th}}>\beta, \\ 0, & \text { elsewhere. }\end{cases}
$$

Accordingly, conditioned on $g_{s p}=\beta$ for $0 \leq \beta \leq I_{\mathrm{th}}$, and due to the considered symmetric scenario, $\mathcal{P}_{L \mid g_{s p}}(\bar{\beta})$ is given in terms of a binomial distribution as

$$
\begin{aligned}
\mathcal{P}_{L \mid g_{s p}}(\beta) & =\left(\begin{array}{c}
K \\
L
\end{array}\right)\left(1-e^{-\frac{I_{\mathrm{th}}-\beta}{\pi_{r p}}}\right)^{L}\left(e^{-\frac{I_{\mathrm{th}}-\beta}{\pi_{r p}}}\right)^{K-L} \\
& =\left(\begin{array}{c}
K \\
L
\end{array}\right) \sum_{l=0}^{L}\left(\begin{array}{c}
L \\
l
\end{array}\right)(-1)^{l} e^{-\frac{(K-L+l)\left(I_{\mathrm{th}}-\beta\right)}{\pi_{r p}}},
\end{aligned}
$$

where the binomial expansion is again utilized. It is straightforward to see that when $\beta>I_{\mathrm{th}}, \mathcal{P}_{L \mid g_{s p}}(\beta)=0$ for $L=1,2, \cdots, K$, while $\mathcal{P}_{L \mid g_{s p}}(\beta)=1$ for $L=0$. Hence, for the case when $L=0$, the support of $\beta$ is $0 \leq \beta \leq \infty$, while it is $0 \leq \beta \leq I_{\text {th }}$ for $1 \leq L \leq K$. Now, we can obtain 


$$
\begin{aligned}
& \left(1-\frac{\pi_{s r} e^{-\alpha\left(\frac{1}{\pi_{s r}}+\frac{\beta+1}{\pi_{r d}}\right)}}{\left(\alpha \pi_{r r}+\pi_{s r}\right)}\right)^{L}=\sum_{l=0}^{L}\left(\begin{array}{l}
L \\
l
\end{array}\right)\left(\frac{-\pi_{s r} e^{-\alpha\left(\frac{1}{\pi_{s r}}+\frac{1}{\pi_{r d}}\right)}}{\left(\alpha \pi_{r r}+\pi_{s r}\right)}\right)^{l} e^{-\frac{\alpha \beta l}{\pi_{r d}}} . \\
& F_{\gamma}^{\mathrm{DL}}(\alpha \mid L)=\sum_{l=0}^{L}\left(\begin{array}{l}
L \\
l
\end{array}\right)\left(\frac{-\pi_{s r} e^{-\alpha\left(\frac{1}{\pi_{s r}}+\frac{1}{\pi_{r d}}\right)}}{\left(\alpha \pi_{r r}+\pi_{s r}\right)}\right)^{l} \frac{\left(1-e^{-\alpha\left(\frac{\alpha l}{\pi_{r d}}+\frac{1}{\pi_{s d}}\right)}\right)}{\left(\alpha l \frac{\pi_{s d}}{\pi_{r d}}+1\right)} .
\end{aligned}
$$

$$
\begin{aligned}
& \mathcal{P}_{L} \text { for } L=1,2, \cdots, K \text { as } \\
& \mathcal{P}_{L}=\int_{0}^{\infty} \mathcal{P}_{L \mid g_{s p}}(\beta) \frac{e^{-\frac{\beta}{\pi_{s p}}}}{\pi_{s p}} \mathrm{~d} \beta=\int_{0}^{I_{\mathrm{th}}} \mathcal{P}_{L \mid g_{s p}}(\beta) \frac{e^{-\frac{\beta}{\pi_{s p}}}}{\pi_{s p}} \mathrm{~d} \beta \\
& =\left(\begin{array}{c}
K \\
L
\end{array}\right) \sum_{l=0}^{L}\left(\begin{array}{c}
L \\
l
\end{array}\right) \frac{(-1)^{l}\left(e^{-\frac{I_{\mathrm{th}}(K-L+l)}{\pi_{r p}}}-e^{-\frac{I_{\mathrm{hh}}}{\pi_{s p}}}\right)}{1-\frac{(K-L+l) \pi_{s p}}{\pi_{r p}}} .
\end{aligned}
$$

For $L=0, \mathcal{P}_{0}$ is given by

$$
\begin{aligned}
\mathcal{P}_{0} & =\int_{0}^{I_{\mathrm{th}}}\left(e^{-\frac{I_{\mathrm{th}}-\beta}{\pi_{r p}}}\right)^{K} \frac{e^{-\frac{\beta}{\pi_{s p}}}}{\pi_{s p}} \mathrm{~d} \beta+\int_{I_{\mathrm{th}}}^{\infty} \frac{e^{-\frac{\beta}{\pi_{s p}}}}{\pi_{s p}} \mathrm{~d} \beta \\
& =\frac{\pi_{r p} e^{-\frac{K I_{\mathrm{th}}}{\pi_{r p}}}-K \pi_{s p} e^{-\frac{I_{\mathrm{th}}}{\pi_{s p}}}}{\pi_{r p}-K \pi_{s p}} .
\end{aligned}
$$

The end-to-end SNR CDF is hence given as

$$
F_{\gamma}^{\mathrm{NDL}}(\alpha)=\sum_{L=0}^{K} F_{\gamma}^{\mathrm{NDL}}(\alpha \mid L) \mathcal{P}_{L},
$$

which is detailed in (29) shown at the top of the next page.

2) With Direct Link: The analysis of $P_{L}$ follows directly from the no direct link case for $L=1,2, \cdots, K$. However, when a direct $\mathrm{s}-\mathrm{d}$ link exists, communication can still take place even when no feasible relays exist. Thus, the event with probability $\mathcal{P}_{0}$ in the no direct link case is now further split into two sub-events. Specifically, communication can still succeed in the sub-event when the sum of the source and relay transmissions do not satisfy the interference constraint for all relays but the source alone does. Let us denote the probability of this sub-event by $\tilde{\mathcal{P}}_{0}$. It is then given by

$$
\tilde{\mathcal{P}}_{0}=\mathbb{P}\left\{\left(\bigcap_{k=1}^{K}\left(g_{k}>I_{\mathrm{th}}\right)\right) \cap\left(g_{s p} \leq I_{\mathrm{th}}\right)\right\} .
$$

Note that $g_{k}$ depends on $g_{s p}$. Nonetheless, $\left\{g_{k}\right\}_{k=1}^{K}$ are mutually independent given $g_{s p}$, and distributed as in (21). Thus, $\tilde{\mathcal{P}}_{0}$ can be written as

$\tilde{\mathcal{P}}_{0}=\int_{\beta=0}^{I_{\mathrm{th}}}\left(e^{-\frac{I_{\mathrm{th}}-\beta}{\pi_{r p}}}\right)^{K} \frac{e^{-\frac{\beta}{\pi_{s p}}}}{\pi_{s p}} \mathrm{~d} \beta=\frac{\pi_{r p}\left(e^{-\frac{K I_{\mathrm{th}}}{\pi_{r p}}}-e^{-\frac{I_{\mathrm{th}}}{\pi_{s p}}}\right)}{\pi_{r p}-K \pi_{s p}}$.

Finally, when $g_{s p}>I_{\text {th }}$, with probability $\mathcal{P}_{0}-\tilde{\mathcal{P}}_{0}=e^{-\frac{I_{\mathrm{th}}}{\pi_{s p}}}$, communication fails resulting in an end-to-end SNR of $\gamma=0$, and hence the distribution is a unit-step function at $\gamma=0$. We can now write the end-to-end SNR CDF as

$$
F_{\gamma}^{\mathrm{DL}}(\alpha)=e^{-\frac{I_{\mathrm{th}}}{\pi_{s p}}}+\left(1-e^{-\frac{\alpha}{\pi_{s d}}}\right) \tilde{\mathcal{P}}_{0}+\sum_{L=1}^{K} F_{\gamma}^{\mathrm{DL}}(\alpha \mid L) \mathcal{P}_{L},
$$

which is detailed in (33) at the top of the next page. It can be verified that (29) is a special case of (33) by setting $\pi_{s d}=0$.

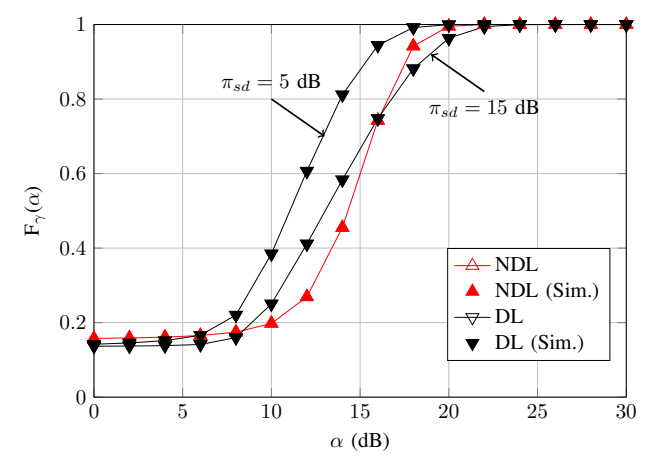

Fig. 2. CDF of end-to-end SNR, for $\pi_{s r}=\pi_{r d}=15 \mathrm{~dB}, \pi_{s d} \in\{5,15\}$ $\mathrm{dB}, \pi_{r r}=-5 \mathrm{~dB}, \pi_{s p}=2 \mathrm{~dB}, \pi_{r p}=3 \mathrm{~dB}, I_{\mathrm{th}}=5 \mathrm{~dB}$, and $K=10$ relays.

It can be also verified that (29) and (33) boil down to (14) and (20) for $L=K$, respectively, in the limit when $I_{\mathrm{th}} \rightarrow \infty$.

\section{NUMERICAL RESUlTS}

In this section, we numerically verify the theoretical findings derived in previous sections. All numerical results are evaluated by averaging over $10^{7}$ sets of channel realizations with the parameters summarized in the caption of each figure. Also, for clarity of presentation, solid lines with unfilled marks are used to plot the theoretical results, while the same filled marks with no connecting lines are used for simulation results. Hence, curves with solid lines and filled marks indicate perfect matching between theoretical and simulation results.

In Fig. 2, the CDF of the end-to-end SNR is plotted for both scenarios with and without secondary direct source-destination link. As shown, since exact performance expressions were derived, the theoretical and simulation results match perfectly. We can notice two observations in the shown figure, namely, a shift in the ordinate and two points of crossover. First, the CDF does not start from zero, which is typically encountered when the analysis involves mixed random variables. Specifically, this shift is due to the unit-step distributions in the events when the end-to-end communication totally fails due to the infeasibility of the relays/source transmissions, thereby yielding an SNR value of zero regardless of the secondary link gains. The event of total communication failure is less probable when a direct link exists, which can be easily noticed from the theoretic expressions, and hence, the shift has a lower value in the scenario with direct link. This shift is mainly controlled by $I_{\text {th }}$, and it decreases as $I_{\text {th }}$ increases. Second, the existing points of crossover between the two curves happen for the fact that the direct link has two contradicting effects; it acts as an interference term in the second hop while it also represents an additional end-to-end path that can be utilized. At low $\pi_{s d}$ values, the direct link is rarely utilized as a beneficial branch, so its adverse effect is clear in the form of a higher probability density of the end-to-end SNR at low SNR values compared to the scenario with no direct link. This is the exact cause 


$$
F_{\gamma}^{\mathrm{NDL}}(\alpha)=\frac{\pi_{r p} e^{-\frac{K I_{\mathrm{th}}}{\pi_{r p}}}-K \pi_{s p} e^{-\frac{I_{t h}}{\pi_{s p}}}}{\pi_{r p}-K \pi_{s p}}+\sum_{L=1}^{K}\left(\begin{array}{l}
K \\
L
\end{array}\right)\left(1-\frac{\pi_{s r} e^{-\alpha\left(\frac{1}{\pi_{s r}}+\frac{1}{\pi_{r d}}\right)}}{\alpha \pi_{r r}+\pi_{s r}}\right) \sum^{L} \sum_{l=0}^{L}\left(\begin{array}{l}
L \\
l
\end{array}\right) \frac{(-1)^{l} \pi_{r p}\left(e^{-\frac{I_{\mathrm{th}}(K-L+l)}{\pi_{r p}}}-e^{-\frac{I_{\mathrm{th}}}{\pi_{s p}}}\right)}{\pi_{r p}-(K-L+l) \pi_{s p}} .
$$

$$
\left.\begin{array}{rl}
F_{\gamma}^{\mathrm{DL}}(\alpha)= & e^{-\frac{I_{t h}}{\pi_{s p}}}+\left(\frac{\pi_{r p}\left(e^{-\frac{K I_{t h}}{\pi_{r p}}}-e^{-\frac{I_{t h}}{\pi_{s p}}}\right)}{\pi_{r p}-K \pi_{s p}}\right)\left(1-e^{-\frac{\alpha}{\pi_{s d}}}\right)+\sum_{L=1}^{K}\left(\begin{array}{l}
K \\
L
\end{array}\right)\left(\sum_{l=0}^{L}\left(\begin{array}{l}
L \\
l
\end{array}\right) \frac{(-1)^{l}\left(e^{-\frac{I_{t h}(K-L+l)}{\pi_{r p}}}-e^{-\frac{I_{t h}}{\pi_{s p}}}\right)}{1-\frac{(K-L+l) \pi_{s p}}{\pi_{r p}}}\right) \\
& \times\left(\sum_{n=0}^{L}\left(\begin{array}{l}
L \\
n
\end{array}\right)\left(\frac{\left.-\pi_{s r} e^{-\alpha\left(\frac{1}{\pi_{s r}}+\frac{1}{\pi_{r d}}\right.}\right)}{\alpha \pi_{r r}+\pi_{s r}}\right)\right. \\
\end{array}\right) .
$$

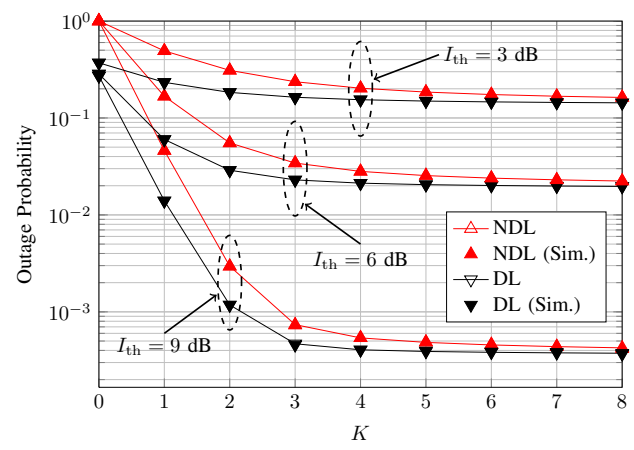

Fig. 3. Outage probability vs. $K$, for $\pi_{s r}=\pi_{r d}=20 \mathrm{~dB}, \pi_{r r}=3 \mathrm{~dB}$, $\pi_{s d}=5 \mathrm{~dB}, \pi_{s p}=0 \mathrm{~dB}, \pi_{r p}=1 \mathrm{~dB}, I_{\mathrm{th}} \in\{3,6,9\} \mathrm{dB}$, and $R=1$ $\mathrm{bit} / \mathrm{sec} / \mathrm{Hz}$

of the leftmost point of crossover. However, for higher $\pi_{s d}$ values, the probability of the direct link to actively participate in the communication as a useful path increases, and hence, its favorable effect shows up. This causes the crossover point at the right of the $\pi_{s d}=15 \mathrm{~dB}$ curve. Depending on the direct link gain, in addition to the channel parameters and number of relays, the crossover may take place once or twice as indicated, and it may never occur for higher direct link gains. The used channel parameters are purposefully selected to show all possible relative behavior in coverage extension versus throughput enhancement scenarios.

When a fixed-rate transmission is adopted, the SNR CDF directly yields the outage probability in the channel. In Fig. 3 , we plot the outage probability versus the number of relays, $K$, with a source rate of $R=1 \mathrm{bit} / \mathrm{sec} / \mathrm{Hz}$, yielding an SNR threshold of $\alpha=2^{R}-1$ below which outage is declared. There are two important observations in this figure that need to be highlighted. First, it is clear that relay selection has a promising potential to boost the performance of the secondary system in underlay networks, in both scenarios of coverage extension and throughput enhancement. Even when a high interference threshold $I_{\text {th }}$ is allowed by the primary user, the performance can be seriously limited for single relay cooperation, while noticeable enhancements can be offered when more relays are available, especially when no direct link exists. Second, a plateau exists as $K$ increases, and its value depends on the interference threshold and the average interference link gains. The average secondary link gains are found to only impact the behavior of how fast this plateau is eventually reached.

\section{CONCLUSion}

In this work, exact performance analysis was performed for opportunistic decode-and-forward full-duplex relay selection, taking the self-interference of the relays into account.
More specifically, the exact cumulative distribution function of the end-to-end signal-to-noise-ratio was derived considering Rayleigh-fading channels. Moreover, the performance of fullduplex relay selection techniques was analyzed in underlay networks where a primary user dictates an interference threshold that cannot be exceeded by the secondary transmissions. The performance is analyzed for both relaying scenarios where either coverage extension or throughput enhancement is targeted. The derived exact analytical results are shown to perfectly match with those obtained via numerical simulations.

\section{REFERENCES}

[1] S. Hong, J. Brand, J. Choi, M. Jain, J. Mehlman, S. Katti, and P. Levis, "Applications of self-interference cancellation in $5 \mathrm{G}$ and beyond," IEEE Commun. Mag., vol. 52, no. 2, pp. 114-121, Feb. 2014.

[2] X. Hong, J. Wang, C.-X. Wang, and J. Shi, "Cognitive radio in 5G: a perspective on energy-spectral efficiency trade-off," IEEE Commun. Mag., vol. 52, no. 7, pp. 46-53, July 2014.

[3] M. Xia and S. Aissa, "Cooperative AF relaying in spectrum-sharing systems: Outage probability analysis under co-channel interferences and relay selection," IEEE Trans. Commun., vol. 60, no. 11, Nov. 2012.

[4] S. I. Hussain, M. M. Abdallah, M.-S. Alouini, M. Hasna, and K. Qaraqe, "Best relay selection using SNR and interference quotient for underlay cognitive networks," in Proc. IEEE ICC'12, Ottawa, Canada, June 2012.

[5] K. B. Fredj and S. Aissa, "Performance of amplify-and-forward systems with partial relay selection under spectrum-sharing constraints," IEEE Trans. Wireless Commun., vol. 11, no. 2, pp. 500-504, Feb. 2012.

[6] J. Lee, H. Wang, J. G. Andrews, and D. Hong, "Outage probability of cognitive relay networks with interference constraints," IEEE Trans. Wireless Commun., vol. 10, no. 2, pp. 390-395, Feb. 2011.

[7] M. Jain, J. Choi, T. Kim, D. Bharadia, S. Seth, K. Srinivasan, P. Levis, S. Katti, and P. Sinha, "Practical, real-time, full duplex wireless," in Proc. ACM MobiCom'11, Las Vegas, NV, Sept. 2011.

[8] M. Duarte, C. Dick, and A. Sabharwal, "Experiment-driven characterization of full-duplex wireless systems," IEEE Trans. Wireless Commun., vol. 11, no. 12, pp. 4296-4307, Dec. 2012.

[9] E. Askari and S. Aissa, "Full-duplex cognitive radio with packet fragmentation," in Proc. IEEE WCNC'14, Istanbul, Turkey, Apr. 2014.

[10] H. Kim, S. Lim, H. Wang, and D. Hong, "Optimal power allocation and outage analysis for cognitive full duplex relay systems," IEEE Trans. Wireless Commun., vol. 11, no. 10, pp. 3754-3765, Oct. 2012.

[11] I. Krikidis, H.A. Suraweera, P. J. Smith, and C. Yuen, "Full-duplex relay selection for amplify-and-forward cooperative networks," IEEE Trans. Wireless Commun., vol. 11, no. 12, pp. 4381-4393, Dec. 2012.

[12] X. Rui, J. Hou, and L. Zhou, "On the performance of full-duplex relaying with relay selection," Electronics Letters, vol. 46, no. 25, pp. 1674-1676, Dec. 2010.

[13] T. Riihonen, S. Werner, and R. Wichman, "Hybrid full-duplex/halfduplex relaying with transmit power adaptation," IEEE Trans. Wireless Commun., vol. 10, no. 9, pp. 3074-3085, Sept. 2011.

[14] T. Kwon, S. Lim, S. Choi, and D. Hong, "Optimal duplex mode for DF relay in terms of the outage probability," IEEE Trans. Veh. Technol., vol. 59, no. 7, pp. $3628-3634$, Sept. 2010. 